BMJ Open

Diabetes

Research

\& Care

\title{
Mealtime insulin BOLUS score increases prior to clinic visits in youth with type 1 diabetes
}

\author{
Andrew McConville (D) , ${ }^{1}$ Amy E Noser, ${ }^{1}$ Mark A Clements, ${ }^{2,3,4}$ Susana R Patton ${ }^{5}$
}

\section{To cite: McConville A,} Noser AE, Clements MA, et al. Mealtime insulin BOLUS score increases prior to clinic visits in youth with type 1 diabetes. BMJ Open Diab Res Care 2020;8:e001348. doi:10.1136/ bmjdrc-2020-001348

Received 10 March 2020 Revised 1 May 2020 Accepted 18 May 2020
Check for updates

(C) Author(s) (or their employer(s)) 2020. Re-use permitted under CC BY-NC. No commercial re-use. See rights and permissions. Published by BMJ.

${ }^{1}$ Clinical Child Psychology Program, University of Kansas, Lawrence, Kansas, USA ${ }^{2}$ Division of Endocrinology, Department of Pediatrics, Children's Mercy Hospitals and Clinics, Kansas City, Missouri, USA

${ }^{3}$ University of Missouri-Kansas City, Kansas City, Missouri, USA ${ }^{4}$ University of Kansas Medical Center, Kansas City, Kansas, USA

${ }^{5}$ Nemours Center for Healthcare Delivery Science, Nemours Children's Health System, Jacksonville, Florida, USA

Correspondence to Dr Susana R Patton; Susana.Patton@nemours.org

\section{ABSTRACT}

Introduction Multiple studies confirm the occurrence of 'white coat adherence' (WCA), a term describing an increase in engagement with self-care tasks just prior to a scheduled clinic appointment, across cohorts with multiple chronic conditions. In youth with type 1 diabetes (T1D), research also shows an increase in self-monitoring blood glucose frequency ahead of youths' clinic visits. While studies show preliminary evidence for the occurrence of WCA in youth with T1D, no study has examined the effect of WCA and mealtime insulin dosing behaviors in youth with T1D. The frequency of mealtime insulin bolusing score (BOLUS) is an objective measure of mealtime insulin use in youth with T1D that could be vulnerable to WCA. To fill this gap in the literature and further our understanding of WCA in pediatric diabetes, we determined whether WCA also impacts BOLUS scores in youth with T1D.

Research design and methods We extracted insulin pump records and $\mathrm{HbA1c}$ levels from a clinical database for 459 youth with $\mathrm{T1D}$ ( $\mathrm{M}_{\mathrm{age}}=12.5 \pm 2.9$ years). We calculated mean BOLUS scores for 6-5, 4-3, and 2-0 weeks prior to youths' routine clinic visits. We used multilevel modeling to examine patterns of BOLUS scores prior to clinic visits and tested for age differences. Results Multilevel modeling showed a significant increase in BOLUS scores in the weeks prior to youths' clinic appointments $(\beta=0.07, p<0.001)$. On average, adolescents had lower BOLUS scores than school-age children $(\beta=-0.35, p<0.001)$. Post hoc analyses showed that adolescents consistently had lower BOLUS scores than children across assessments (p's $<0.001$ ).

Conclusions Youth with T1D increase their mealtime insulin use prior to clinic appointments. The BOLUS may be a viable target for intervention to drive improved glycemic control. Whether increased tendency to WCA is associated with reduced risk of diabetic complications remains to be determined.

\section{INTRODUCTION}

Many youth with type 1 diabetes (T1D) do not complete self-management tasks consistently across all days between clinic visits. 'White coat adherence' (WCA) is a term that describes an increase in self-management behaviors just prior to a scheduled clinic appointment. ${ }^{1}$ Studies show that WCA occurs in both adult and pediatric populations and affects a variety of chronic illness groups. ${ }^{2-5}$

\section{Significance of this study}

What is already known about this subject?

- Research shows evidence for 'white coat adherence' (WCA) in youth with type 1 diabetes (T1D) pertaining to frequency of self-monitoring blood glucose.

- Specifically, recent studies found youth with T1D engage in more self-monitoring just before a clinic appointment.

What are the new findings?

- Our findings demonstrated that the mealtime insulin BOLUS score, a validated and objective measure of insulin use, is also impacted by WCA.

- BOLUS scores increased significantly in the 6 weeks leading up to youths' scheduled clinic appointments, for both children and adolescents.

How might these results change the focus of research or clinical practice?

- Diabetes care teams and researchers should consider using insulin pump downloads from up to 6 weeks prior to clinic appointments to examine more representative patterns of mealtime insulin use.

- Our findings highlight the effect of WCA as a potential target for future clinical intervention, in which youth with T1D may see clinical benefits from more frequent contact in the period between their clinic appointments.

In two recent studies, researchers confirmed the occurrence of WCA in youth with T1D. Specifically, these studies found that youth engaged in more frequent blood glucose monitoring in the weeks just prior to clinic visits, but there were mixed results for insulin use. ${ }^{26}$ We previously developed the mealtime insulin BOLUS score as an objective measure of mealtime insulin use in youth with T1D using insulin pump data and confirmed its validity by associating youth's BOLUS scores with current and future glycated hemoglobin (HbA1c) levels. $^{78}$ We also found BOLUS scores were better able to explain variability in $\mathrm{HbA1c}$, thus highlighting the utility of BOLUS scores in describing T1D 
self-management behaviors. Whether BOLUS scores are impacted by WCA remains unknown.

It is important to determine whether WCA impacts mealtime insulin bolus behaviors because clinicians typically guide insulin adjustment based on historic selfmanagement data proximal to a clinic visit $(0-2$ weeks prior). Yet, WCA can mask suboptimal self-management behaviors at times distal to an upcoming clinic visit. If providers do not witness evidence of suboptimal selfmanagement, they may fail to offer corrective treatment guidance or deliver needed behavioral interventions. Finally, if research demonstrates that WCA influences HbAlc or average glycemia, then one could hypothesize that behavioral interventions involving more frequent contact with the clinician or care team might be more effective than similar interventions with less frequent contact.

We sought to determine whether WCA impacts mealtime insulin BOLUS scores in a sample of youth with T1D. Specifically, we tested the hypothesis that BOLUS scores would increase just prior to routine clinic visits, suggesting the occurrence of WCA. We also examined whether children and adolescents differ in their BOLUS scores prior to routine clinic visits, which might suggest differences in WCA effects for the BOLUS as a function of child age.

\section{SUBJECTS AND METHODS}

We identified eligible youth from the previously described Mercy on $T O_{D} P$ health outcomes database, ${ }^{9} 10$ which now contains over 7000 patient records and extracted demographics (ie, child age, race/ethnicity, duration of T1D), values for HbA1c, and 6 weeks of insulin pump records from the most recent clinic appointments. Inclusion criteria for participants in the present study included (1) child age between 7 and 19 years old, (2) a T1D diagnosis of at least 1 year, (3) daily insulin pump use, and (4) at least one clinic visit with 6 weeks of insulin pump data available in the database. These criteria yielded 459 eligible youth.

\section{Data collection}

We collected insulin pump data in three 14-day blocks $(6-5,4-3$, and $2-0$ weeks) prior to a youth's routine clinic visit. We further collected youth's demographic information as well as HbAlc levels from the corresponding clinic visit.

\section{Measures}

Mealtime insulin BOLUS

The BOLUS provides an objective measure of engagement with mealtime insulin dosing. Specifically, the BOLUS score is calculated on an integer scale (0-3) by tallying the presence of at least one carbohydrateassociated bolus during the time periods 06:00-10:00, 11:00-15:00 and 16:00-22:00 daily. The measure is reported as a 14-day average of daily scores, with higher scores indicating more frequent mealtime bolusing. ${ }^{7} \mathrm{We}$ calculated the mean daily BOLUS score for youth within each of the three monitoring periods (ie, weeks 6-5, 4-3, and $2-0$ before the clinic visit).

\section{Youth $\mathrm{HbA1c}$}

Youth's HbA1c values came from clinical management using the Tosoh G8 HPLC Analyzer (Tosoh, San Francisco, CA, USA) or the Afinion Point of Care Analyzer (Abbott Laboratories, Chicago, IL, USA). These are both reliable methods of measuring HbAlc that are traceable to Diabetes Control and Complications standards. ${ }^{11} 12$

\section{Analyses}

We used multilevel modeling to examine patterns of BOLUS scores prior to youth's clinic visits. We used multilevel modeling to account for repeated measures that were nested within a participant and sample sizes across monitoring periods. ${ }^{13-15}$ We specified change over time as a function of time before clinic visit using the previously described monitoring periods (ie, weeks $6-5$, $4-3$, and 2-0 before the clinic visit). We centered time before clinic visit at weeks $6-5$ before the clinic visit. We analyzed age differences in models by using a dichotomous variable for youth age status. For these moderation analyses, child ( $<13$ years old) was coded as 0 and adolescent ( $\geq 13$ years old) was coded as 1 . We conducted all analyses using SAS PROC MIXED (SAS V.9.4) and used the -2 restricted log likelihood (-2LL) to examine the best fitting model for time. We estimated all models using the restricted maximum likelihood estimator and used $\mathrm{p}$ values $<0.05$ to determine statistical significance.

\section{RESULTS}

\section{Sample}

Youth in our sample had a mean age of $12.5 \pm 2.9$ years, a mean duration of T1D of $4.6 \pm 3.5$ years, and a mean HbA1c of $8.4 \% \pm 1.6 \%$. There were 199 adolescents (aged $\geq 13$ ) and 260 children in our sample and the mean BOLUS score across monitoring periods was $2.15 \pm 0.61$.

\section{Trajectory of BOLUS scores}

The intraclass correlation for BOLUS scores was 0.763, suggesting that $76.3 \%$ of the variance in BOLUS scores was between persons. The best fitting model was a random linear time model; the addition of random linear time significantly improved model fit, $-2 \Delta \operatorname{LL}(\sim 2)=19.1$, $\mathrm{p}<0.0001$. Overall, we found that time was a significant predictor of BOLUS scores $(\beta=0.054, \mathrm{SE}=0.013$, $\mathrm{p}<0.001$ ), such that BOLUS scores increased in the weeks leading up to youth's clinic visits. Figure 1 presents the change in BOLUS scores across each monitoring period for the total sample. Next, we examined the effect of youth age status (ie, child vs adolescent) at baseline and its interaction with time. Results showed significant main effects of time $(\beta=0.068, \mathrm{SE}=0.018, \mathrm{p}<0.001)$ and age status $(\beta=-0.35, \mathrm{SE}=0.06, \mathrm{p}<0.001)$. However, the interaction of time by age status was not significant $(\beta=-0.032$, $\mathrm{SE}=0.027, \mathrm{p}=0.23$ ), indicating that rate of change for 


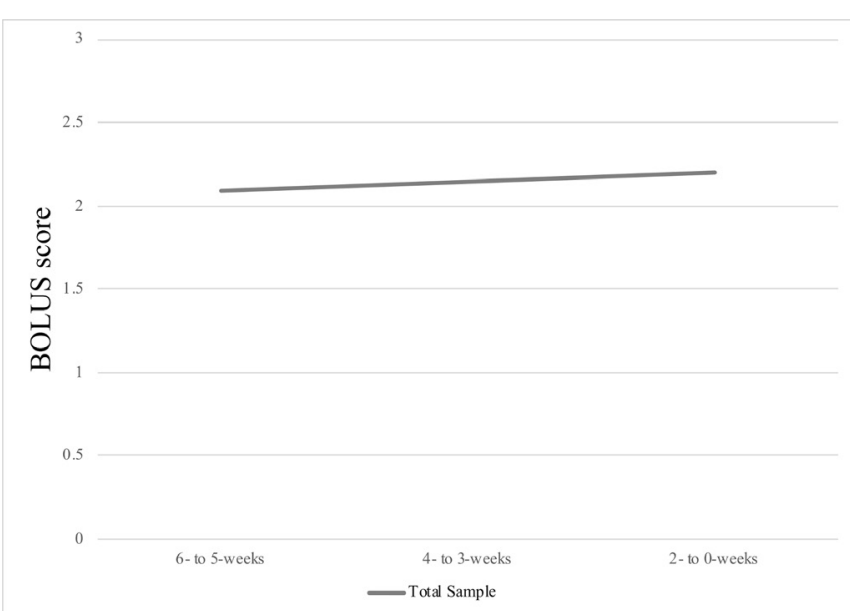

Figure 1 BOLUS scores across monitoring periods for total sample.

BOLUS scores did not differ based on age status. Post hoc tests showed children had higher BOLUS scores than adolescents at each monitoring period ( $6-5$ weeks $(\beta=0.322, \quad \mathrm{SE}=0.063, \mathrm{p}<0.0001), \quad 4-3$ weeks $\quad(\beta=0.406$, $\mathrm{SE}=0.056, \mathrm{p}<0.0001)$, and $2-0$ weeks before clinic visit $(\beta=0.404, \mathrm{SE}=0.055, \mathrm{p}<0.0001))$. Figure 2 presents the change in BOLUS across each monitoring period for children and adolescents. Table 1 presents model estimates for BOLUS scores for the entire sample as well as subgroups of children and adolescents.

\section{DISCUSSION}

This study extends existing research on both WCA and the BOLUS score in youth with T1D by investigating the impact of WCA on mealtime insulin BOLUS scores. In support of our primary hypothesis, our results indicated that youth BOLUS scores appeared to increase in the weeks prior to routine clinic visits. These findings are consistent with previous studies illustrating the impact of WCA on frequency of self-monitoring blood glucose in youth with T1D. ${ }^{26}{ }^{16}$ When examining

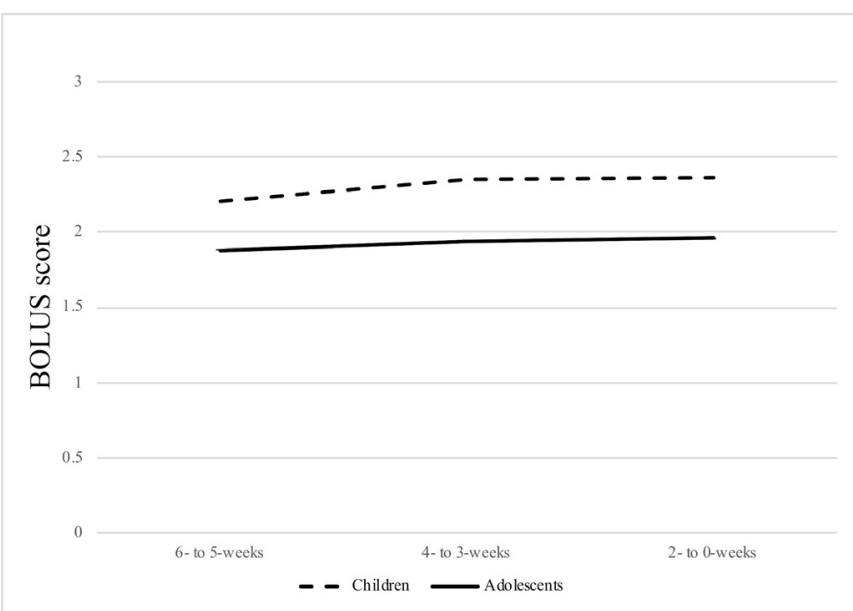

Figure 2 BOLUS scores across monitoring periods for children versus adolescents.

\begin{tabular}{llll} 
Table 1 & BOLUS scores across assessment points \\
\hline & $\begin{array}{l}\text { BOLUS } \\
\text { score }\end{array}$ & $\begin{array}{l}\text { BOLUS } \\
\text { score }\end{array}$ & $\begin{array}{l}\text { BOLUS } \\
\text { score }\end{array}$ \\
Group & $\mathbf{6 - 5}$ weeks & $\mathbf{4 - 3}$ weeks & $\mathbf{2 - 0}$ weeks \\
\hline $\begin{array}{l}\text { Total sample, } \\
\text { estimate (SE) }\end{array}$ & $2.09(0.031)$ & $2.14(0.027)$ & $2.20(0.029)$ \\
Children & $2.20(0.043)$ & $2.35(0.037)$ & $2.36(0.036)$ \\
Adolescents & $1.88(0.046)$ & $1.94(0.042)$ & $1.96(0.042)$ \\
\hline
\end{tabular}

developmental differences (ie, children vs adolescents) on the effect of WCA, we found that adolescents consistently had significantly lower BOLUS scores than children across each time period. Our results showed that adolescents had BOLUS scores of 0.3-0.4 points lower than children across each time period; a difference which translates to nearly five more missed mealtime boluses for adolescents in each 2 weeks period relative to that for children. However, we did not find an interaction between time and age status for youth's BOLUS scores. That is, overall, children and adolescents both missed an average of 1.5 mealtime boluses more in weeks 6 and 5 prior to their clinic visit than in weeks 2 and 1 prior to their clinic visit. In our validation of the BOLUS method, our data suggested that a 1 point increase in youth BOLUS scores may translate into a $1.5 \%$ decrease in youth $\mathrm{HbAlc}$, underscoring how closely the BOLUS can relate to youth's glycemic control. ${ }^{7}$ Therefore, our current data also suggest that the seemingly lower BOLUS score in weeks 6 and 5 before youth's clinic visit may relate to a clinically significant effect on their HbAlc and overall health.

In the present cohort, youth on average increased their BOLUS scores as the date of their clinic visit approached. It is possible that families did this in anticipation of receiving motivation and treatment guidance from their diabetes care team. However, increasing mealtime insulin use, even if only for a few weeks, can help youth to achieve tighter blood glucose levels and may offer longer term benefits in reducing youth's risk of T1D-related complications. ${ }^{17} 18$ One could hypothesize, therefore, that clinicians could leverage the WCA effect for mealtime insulin use to achieve a clinical benefit. Namely, youth with T1D may receive potential value from more frequent clinic appointments or from regular data sharing accompanied by contact with the youth's diabetes care team between clinic visits. Measuring engagement via the BOLUS score accurately and continuously might also support improved T1D outcomes by enabling the creation of 'risk' alerts to healthcare providers, who could in turn intensify behavioral treatment approaches to optimize self-management.

One disadvantage of the WCA effect is that it may give a false impression of a youth's average daily level of T1D self-management if diabetes care teams and researchers only download and review insulin pump data for the 
2 week period immediately before the youth's clinic visit. Specifically, 2-week downloads could overestimate youth's typical mealtime insulin use and lead diabetes care teams to make a potentially suboptimal change to youth's insulin dosage. Thus, our results also suggest that diabetes care teams should consider using insulin pump downloads from up to 6 weeks prior to youth's clinic visit to enable them to examine the dynamically changing pattern of youth's BOLUS scores and to make changes in insulin dosages that consider potential WCA effects.

The strengths of our study include its longitudinal design, large sample size, and objectively measured T1D self-management behavior (ie, BOLUS scores). However, we also acknowledge several limitations. One limitation is that our study only examined T1D engagement for mealtime insulin use in youth using insulin pumps. We would assert that this is only a minor limitation because recent data from the T1 Diabetes Exchange suggest that $63 \%$ of youth with T1D in the USA use an insulin pump. ${ }^{19}$ Another limitation is that our study used observational data collected retrospectively from patient records in a research repository; a prospective data collection would have also allowed us to perform hypothesis-driven quantitative psychological or behavioral assessments. Therefore, we are limited in our ability to describe any factors that relate to our WCA effects and we cannot report on factors that could have caused the WCA effects. A third limitation is that our study used clinical data from a Midwestern single pediatric diabetes center in the USA that serves patients who are primarily non-Hispanic White and middle to upper middle class. Consequently, we must acknowledge that our results may not generalize to more diverse racial, ethnic, or socioeconomic samples. Finally, we acknowledge a fourth limitation related to our observation period, which we limited to 6 weeks before a youth's clinic visit. It is possible that our results here only reveal part of youth's pattern of mealtime insulin use and that future research should consider calculating youth BOLUS scores continuously between clinic visits to better track possible WCA effects.

Our study showed WCA effects for youth's BOLUS scores in the weeks prior to their routine clinic visits for T1D regardless of youth age. Because adolescents had significantly lower BOLUS scores than children across each time period, our WCA effects may be particularly clinically noteworthy when caring for adolescents. A practical implication of our study is the potential value in measuring youth BOLUS scores for up to 6 weeks prior to the youth's clinic visit when interpreting these data in clinical management or research. Future studies should assess patterns in WCA for time periods greater than 6 weeks prior to youth's routine diabetes clinic visit and should also consider whether WCA effects could be further influenced by the time of year (eg, summer) or sociodemographic factors. Finally, future clinical trials should examine for WCA effects in youth receiving more intensive experimental interventions and determine if more frequent contact with diabetes care teams could help stabilize youth's mealtime bolus patterns and help youth to achieve more optimal glycemic control.

Acknowledgements We thank Mitchell Barnes for demographic and HbA1c data collection.

Contributors AM and SRP wrote the paper. SRP designed the research study. AEN analyzed the data. MAC helped design the research study and provided feedback on the paper.

Funding This research was supported in part by grant DK 100779 (to SRP) from the National Institute of Diabetes and Digestive and Kidney Diseases, National Institutes of Health.

Competing interests MAC is the chief medical officer for Glooko and reports personal fees from Eli Lilly and Medtronic, outside the submitted work.

\section{Patient consent for publication Not required.}

Ethics approval This study was approved by the Children's Mercy Hospital Institutional Review Board (IRB number 11120355). Data collection and analyses were approved by the local institutional review board.

Provenance and peer review Not commissioned; externally peer reviewed.

Data availlability statement Data are available upon reasonable request.

Open access This is an open access article distributed in accordance with the Creative Commons Attribution Non Commercial (CC BY-NC 4.0) license, which permits others to distribute, remix, adapt, build upon this work non-commercially, and license their derivative works on different terms, provided the original work is properly cited, appropriate credit is given, any changes made indicated, and the use is non-commercial. See: http://creativecommons.org/licenses/by-nc/4.0/.

ORCID iD

Andrew McConville http://orcid.org/0000-0001-5221-0964

\section{REFERENCES}

1 Cramer JA, Scheyer RD, Mattson RH. Compliance declines between clinic visits. Arch Intern Med 1990;150:1509.

2 Driscoll KA, Wang Y, Bennett Johnson S, et al. White coat adherence in pediatric patients with type 1 diabetes who use insulin pumps.

J Diabetes Sci Technol 2016;10:724-9.

3 Podsadecki TJ, Vrijens BC, Tousset EP, et al. "White coat compliance" limits the reliability of therapeutic drug monitoring in HIV-1-infected patients. HIV Clin Trials 2008:9:238-46.

4 Modi AC, Ingerski LM, Rausch JR, et al. White coat adherence over the first year of therapy in pediatric epilepsy. $J$ Pediatr 2012:161:695-9.

5 Krejci-Manwaring J, Tusa MG, Carroll C, et al. Stealth monitoring of adherence to topical medication: adherence is very poor in children with atopic dermatitis. J Am Acad Dermatol 2007;56:211-6.

6 Driscoll KA, Johnson SB, Tang Y, et al. Does blood glucose monitoring increase prior to clinic visits in children with type 1 diabetes? Diabetes Care 2011;34:2170-3.

7 Patton SR, Clements MA, Fridlington A, et al. Frequency of mealtime insulin bolus as a proxy measure of adherence for children and youths with type 1 diabetes mellitus. Diabetes Technol Ther 2013;15:124-8.

8 Patton SR, DeLurgio SA, Fridlington A, et al. Frequency of mealtime insulin bolus predicts glycated hemoglobin in youths with type 1 diabetes. Diabetes Technol Ther 2014;16:519-23.

9 Raman S, Dai H, DeLurgio SA, et al. High hemoglobin A1c variability is associated with early risk of microalbuminuria in children with T1D. Pediatr Diabetes 2016;17:398-406.

10 Clements MA, Lind M, Raman S, et al. Age at diagnosis predicts deterioration in glycaemic control among children and adolescents with type 1 diabetes. BMJ Open Diabetes Res Care 2014;2:e000039.

11 Lenters-Westra E, Slingerland RJ. Three of 7 hemoglobin a1c point-of-care instruments do not meet generally accepted analytical performance criteria. Clin Chem 2014;60:1062-72.

12 National Glycohemoglobin Standardization Program. List of NGSP certified methods, 2020. Available: http://www.ngsp.org/certified.asp [Accessed 1 Feb 2020]. 
13 Hoffman L. Longitudinal analysis: modeling within-person fluctuation and change. New York, NY: Routledge/Taylor \& Francis Group, 2015.

14 Raudenbush SW, Bryk AS. Hierarchical linear models: applications and data analysis methods. 2nd edn. London, UK: Sage, 2002.

15 Snijders T, Bosker RJ. Multilevel analysis: an introductionto basic and applied multilevel analysis. 2nd edn. London, UK: Sage, 2012.

16 Driscoll KA, Wang Y, Johnson SB, et al. White coat adherence occurs in adolescents with type 1 diabetes receiving intervention to improve insulin pump adherence behaviors. J Diabetes Sci Technol 2017;11:455-60.
17 Prahalad P, Tanenbaum M, Hood K, et al. Diabetes technology: improving care, improving patient-reported outcomes and preventing complications in young people with type 1 diabetes. Diabet Med 2018;35:419-29.

18 Burdick J, Chase HP, Slover RH, et al. Missed insulin meal boluses and elevated hemoglobin $\mathrm{A} 1 \mathrm{c}$ levels in children receiving insulin pump therapy. Pediatrics 2004;113:e221-4.

19 Foster NC, Beck RW, Miller KM, et al. State of type 1 diabetes management and outcomes from the T1D exchange in 2016-2018. Diabetes Technol Ther 2019;21:66-72. 